\title{
THE
}

1989

\section{Locating Stationary Paths in Functional Integrals: An Optimization Method Utilizing the Stationary Phase Monte Carlo Sampling Function}

\author{
Thomas L. Beck \\ Jimmie D. Doll \\ David L. Freeman \\ University of Rhode Island, dfreeman@uri.edu
}

Follow this and additional works at: https://digitalcommons.uri.edu/chm_facpubs

Terms of Use

All rights reserved under copyright.

\section{Citation/Publisher Attribution}

Beck, T. L., Doll, J. D., \& Freeman, D. L. (1989). Locating Stationary Paths in Functional Integrals: An Optimization Method Utilizing the Stationary Phase Monte Carlo Sampling Function. J. Chem. Phys., 90(6), 3181-3191. doi: 10.1063/1.455868

Available at: http://dx.doi.org/10.1063/1.455868

This Article is brought to you for free and open access by the Chemistry at DigitalCommons@URI. It has been accepted for inclusion in Chemistry Faculty Publications by an authorized administrator of DigitalCommons@URI. For more information, please contact digitalcommons-group@uri.edu. 


\title{
Locating stationary paths in functional integrals: An optimization method utilizing the stationary phase Monte Carlo sampling function
}

\author{
Thomas L. Beck and J. D. Doll \\ Los Alamos National Laboratory, MS G-738, Los Alamos, New Mexico 87545 \\ David L. Freeman \\ Department of Chemistry, University of Rhode Island, Kingston, Rhode Island 02881
}

(Received 6 September 1988; accepted 17 November 1988)

\begin{abstract}
A method is presented for determining the stationary phase points for multidimensional path integrals employed in the calculation of finite-temperature quantum time correlation functions. The method can be used to locate stationary paths at any physical time; in the case that $t \gg \beta \hbar$, the stationary points are the classical paths linking two points in configuration space. Both steepest descent and simulated annealing procedures are utilized to search for extrema in the action functional. Only the first derivatives of the action functional are required. Examples are presented first of the harmonic oscillator for which the analytical solution is known, and then for anharmonic systems, where multiple stationary phase points exist. Suggestions for Monte Carlo sampling strategies utilizing the stationary points are made. The existence of many and closely spaced stationary paths as well as caustics presents no special problems. The method is applicable to a range of problems involving functional integration, where optimal paths linking two end points are desired.
\end{abstract}

\section{INTRODUCTION}

The use of Monte Carlo methods in conjunction with a path-integral formulation of quantum mechanics has created a new tool in the calculation of equilibrium and dynamical properties of quantum many-body systems. ${ }^{1-4}$ The calculation of time-dependent quantities for multidimensional quantum systems viā such a method proves to be a difficult task because of the highly oscillatory nature of the integrands. This behavior occurs because the time propagator $\exp (i H t / \hbar)$ leads to integrals involving complex exponentials, exponentials which become more and more oscillatory as the physical time $t$ increases. This is in contrast to the equilibrium case where the density matrix elements of interest are purely real and the associated calculations are amenable to standard Monte Carlo procedures.

Several recent developments, however, have led to an increased optimism concerning the utility of Monte Carlo methods in the evaluation of integrals arising in the calculation of real time properties. ${ }^{5-13}$ Recently, we have presented an extension of traditional equilibrium Monte Carlo techniques aimed at evaluating high-dimensional averages of rapidly oscillating integrands, the stationary phase Monte Carlo (SPMC) method. ${ }^{6,8}$ The basic theory as well as examples of its applications to prototype problems and to the calculation of real time, finite-temperature correlation functions are described elsewhere. ${ }^{9}$

In the SPMC method, numerical difficulties associated with the Monte Carlo construction of averages of highly oscillatory integrands are treated by means of a "coarse graining" procedure. In essence, the method amounts to devising a "filtering" strategy for identifying and removing unnecessary "noise" from the integrand. This approach can be made exact, both formally and in practice. In situations where the integrands are highly oscillatory, the method results in a substantial increase in computational efficiency over stan- dard Monte Carlo evaluations. For the important case of the calculation of finite-temperature correlation functions, the SPMC approach permits studies at times well beyond the thermal time $\beta \hbar$. Connections between our approach and the work by Filinov ${ }^{10}$ and Makri and Miller ${ }^{12,13}$ are described elsewhere. ${ }^{8}$

The SPMC method tends to emphasize regions of configuration space where the action functional is stationary, i.e., extrema of the action, while filtering out regions of rapid oscillations. For the case of a pure propagator, the stationary points of the action for paths connecting two points in configuration space correspond to the classical paths linking these two points. However, when averages are calculated at finite temperature, for example, in the calculation of finitetemperature time correlation functions, the stationary points reflect both equilibrium and dynamical considerations.

In this paper we present an optimization method which utilizes the SPMC technique coupled to steepest descent quench and simulated annealing procedures to search for the stationary points of the multidimensional action functionals. The purpose of this work is twofold. First, locating the stationary points of the multidimensional path integrals is important in understanding the sampling issues involved in the fully quantum-mechanical Monte Carlo calculation of the integrals, since the Metropolis Monte Carlo sampling method must cover all of the dynamically important portions of the configuration space on a reasonable time scale. Knowledge of the number and location of the stationary points of the action gives some indication of the complexity of the integral.

Second, once the stationary paths are located for a given density matrix element, the paths can be incorporated into a Monte Carlo procedure where the Metropolis walk samples fluctuations about each of the stationary paths with periodic random additions of vectors linking the stationary regions. 
This sampling strategy avoids the possibility of a large number of separated stationary phase regions which are not accessed during the course of the simulation. Cline and Wolynes $^{14}$ have discussed a similar method where fully quantum results are obtained for a dissipative system by decorating classical paths with quantum fluctuations. We perform calculations to locate the stationary points in a variety of examples and propose a Monte Carlo strategy for sampling the stationary regions.

In a more general context, the optimization procedure discussed below can be used to locate optimal paths for processes that link two end points, for example, in a functional integral representation of a nonequilibrium thermodynamic process. ${ }^{15}$ The optimization method may also be useful, for example, in determining least energy reaction paths for multidimensional systems where the starting and final configurations are known, and the path linking the initial and final configurations is represented by a Fourier expansion. ${ }^{16}$

The present paper is concerned with a Monte Carlo path integral method for determining and then using the stationary paths in a fully quantum Monte Carlo procedure for the calculation of time correlation functions at finite temperature. The method avoids the time consuming calculation of second derivative matrices with subsequent determinant calculation and diagonalization common to traditional stationary phase approximations to path integrals ${ }^{17,18}$ and other Monte Carlo strategies. ${ }^{12,13}$ It is thus numerically feasible in applications to highly nonlinear multidimensional quantum problems where many classical paths may connect two points in configuration space or where caustics may occur.

We will not discuss semiclassical procedures involving wave-packet methods and/or classical trajectory calculations. ${ }^{19-23}$ These methods generally invoke trajectory calculations in real or imaginary time (classically forbidden processes) or complex phase space coordinates. Typically, one must find all trajectories that connect the desired initial and final states. The path integral prescription fixes the end points and searches for all the paths linking the end points which lead to stationary (or near stationary) points in the action. Wandzura, ${ }^{24}$ Klauder, ${ }^{25}$ and Zhang, Levy, and Friesner ${ }^{26}$ have recently discussed useful methods for approximate evaluations of the path integral.

The paper is organized as follows. In Sec. II we present a short summary of the stationary phase Monte Carlo method. The steepest descent quench and simulated annealing procedures for the location of stationary points are discussed in Sec. III. The application of the method to some simple harmonic and anharmonic examples is described in Sec. IV, with discussion of the results and suggestions for a Monte Carlo sampling strategy given in Sec. V.

\section{STATIONARY PHASE MONTE CARLO}

In this section we present a short summary of the stationary phase Monte Carlo method. More detailed discussions of the method appear elsewhere. ${ }^{8,9}$ For present purposes, it is sufficient to consider a prototype one-dimensional integral of the form

$$
I(t)=\int d x \rho(x) e^{i f f(x)}
$$

The quantity $\rho(x)$ is a positive-definite weight function which will be associated with a thermal weight in the applications to the calculation of finite-temperature time correlation functions discussed below, and $f(x)$ is a phase function associated with the dynamics in the applications. The method summarized below is readily extended to multidimensional applications. The integral can be rewritten as

$$
I(t)=\int d x \rho(x) D(x) e^{i t f(x)},
$$

where $D(x, t)$ is given by

$$
D(x)=\int d y P(y) \frac{\rho(x-y)}{\rho(x)} e^{i t[f(x-y)-f(x)]} .
$$

This identity holds as long as $P(y)$ is a normalized probability distribution (typically chosen to be a prelimit delta function) and the integration is performed over either an infinite $y$ interval or an interval where $\rho$ and the phase function are periodic. The function $D(x, t)$ acts as a "damping function" which filters out the highly oscillatory regions of the integrand. This behavior can be observed by considering the first-order gradient expansion approximation to the damping function. Let $P(y)$ be a Gaussian function with width $\epsilon$. The length scale of $\epsilon$ is chosen to optimally filter the integrand in oscillatory regions away from the stationary phase points. ${ }^{8}$ Then the first-order expression $D_{0}(x, t)$ is given by

$$
D(x) \cong D_{0}(x) \equiv \exp \left[-\left(\epsilon t f^{\prime}\right)^{2} / 2\right],
$$

indicating that this function damps regions of the integrand where the phase function is not stationary. The first-order gradient form of the damping function leads to an approximate evaluation of the integral, but can be made exact by the inclusion of corrections via a secondary Monte Carlo procedure for the calculation of $D(x, t)$, given by Eq. $(2.3){ }^{8,9}$

By including this first-order gradient expression for the damping function in the integral as follows:

$$
I(t)=\int d x \rho(x) D_{0}(x)\left[\frac{D(x)}{D_{0}(x)}\right] e^{i f f(x)},
$$

one can set up a sampling scheme for the Monte Carlo integration which uses the product of $\rho$ and $D_{0}$ as the dynamical importance sampling (DIS) function. This sampling function then contains both thermal and dynamical information, since $\rho(x)$ is the thermal weight function and $D_{0}(x)$ is a function of the first derivative of the phase function, which involves dynamical properties.

This Monte Carlo procedure emphasizes the portions of configuration space where the phase function is stationary and yet "thermally accessible" by the $\rho$ distribution. We will see below that some stationary points in the phase function may lead to thermal weights that are negligible. Also, for an integral involving nonlinear terms in the phase function, multiple stationary phase points result, and then the Monte Carlo procedure becomes one of sampling appropriately over all of the relevant parts of the configuration space in a way that is computationally feasible. It is thus important to first find and then ensure that the Metropolis walk samples all of these relevant portions of the configuration space.

In Ref. 9, we used the general SPMC method described above to calculate real time correlation functions at finite 
temperature for a one-dimensional harmonic oscillator and for several anharmonic one-dimensional examples. The derivation of the finite-temperature time correlation function in the Fourier language is given in Ref. 9. The structure of the multidimensional path integral for the time correlation function is formally the same as the one-dimensional example discussed above. That is, the thermally symmetrized correlation function $G_{A B}(t)$ can be written as

$$
G_{A B}(t)=\frac{\int d x d x^{\prime} d \mathbf{a} d \mathbf{b} \rho([a],[b]) \exp [i \tau f([\mathbf{a}],[\mathbf{b}])] A(x) B\left(x^{\prime}\right)}{\int d x d x^{\prime} d \mathbf{a} d \mathbf{b} \rho([\mathbf{a}],[\mathbf{b}]) \exp [i \tau f([\mathbf{a}],[\mathbf{b}])]}
$$

where $x$ and $x^{\prime}$ refer to the configuration space end points of the path and the $a$ and $b$ refer to the sets of Fourier coefficients used to parametrize the path linking $x$ and $x^{\prime}$ and the return path from $x^{\prime}$ to $x$, respectively. The variable $\tau$ is the ratio of the physical time to the symmetrized thermal time, i.e., $\tau=t /(\beta \hbar / 2)$. Each path is expanded in a Fourier series about a straight line reference path as follows:

$$
\begin{aligned}
& x_{\mathrm{a}}(u)=x+\left(x^{\prime}-x\right) u+\sum_{k} a_{k} \sin (k \pi u), \\
& x_{\mathrm{b}}(u)=x^{\prime}+\left(x-x^{\prime}\right) u+\sum_{k} b_{k} \sin (k \pi u) .
\end{aligned}
$$

See Fig. 1 for a schematic representation of the paths. The quantity $\rho([a],[b])$ is given by

$$
\rho([\mathbf{a}],[\mathbf{b}])=\left|\rho_{\mathrm{fp}}\left(x^{\prime}, x, \beta_{c}\right)\right|^{2} e^{-\left[S_{+}([\mathbf{a}])+S_{+}([\mathbf{b}])\right]},
$$

where $\rho_{\mathrm{fp}}$ is the free particle weight and the phase function is

$$
f([\mathbf{a}],[\mathbf{b}])=S_{-}([\mathbf{a}])-S_{-}([\mathbf{b}]) .
$$

$S_{ \pm}$is given by

$$
S_{ \pm}([\mathrm{a}])=\sum_{k} a_{k}^{2} / 2 s_{k}^{2} \pm \frac{\beta}{2} \int_{0}^{1} V\left(x_{\mathrm{a}}(u)\right) d u .
$$

The Gaussian width appearing in (2.10) is given by

$$
s_{k}^{2}=2\left|\beta_{c}\right|^{2} \hbar^{2} /\left[m \pi^{2} k^{2}(\beta / 2)\right],
$$

with $\beta_{c}=\beta / 2+i t / \hbar$.

The $\rho$ ( [a], [b] ) portion of the integral corresponds to a thermal weight which has the structure of the exponential of a Hamiltonian (or, more appropriately, a Lagrangian in imaginary time) integrated along a given path while the

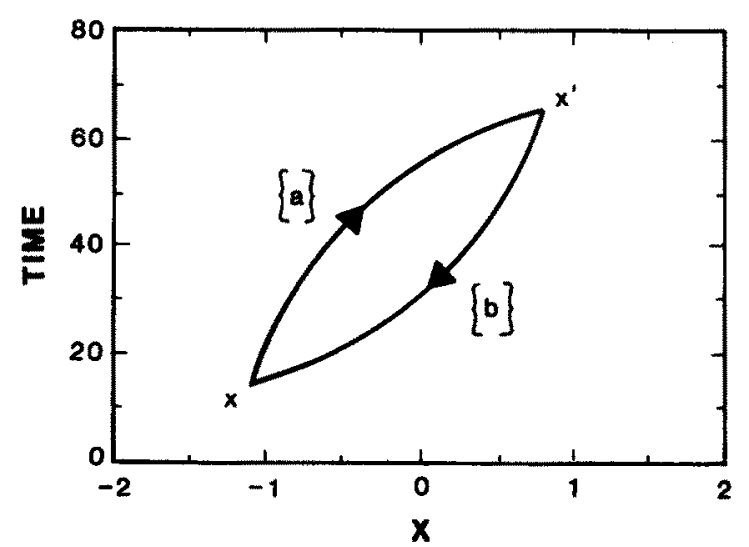

FIG. 1. Schematic representation of the outgoing path linking $x$ and $x^{\prime}$ parametrized by the a set of coefficients, and the return path parametrized by the $b$ set of coefficients. The Monte Carlo integration of the path integral involves sampling over all such end-point configurations, $x$ and $x^{\prime}$, and the two sets of Fourier coefficients. phase function has the structure of a Lagrangian integrated along the path. It is the stationary points of the $S_{-}$functional that determine which paths are most important in the calculation of the path integral, as long as the paths are thermally accessible. Notice that for physical times $t \geqslant \beta \hbar$, the action functional (phase function) takes on the form observed in a pure propagator. It is in this limit that the stationary phase points can be associated with classical paths linking the end points.

The first-order gradient expression for the damping function in this multidimensional example is given by

$$
\begin{aligned}
& D_{0, \epsilon}([\mathbf{a}],[\mathbf{b}]) \\
& =\exp \left(-\frac{1}{2} \sum_{k=1} \tau^{2} \epsilon_{k}^{2}\left\{\left[\partial f([\mathbf{a}],[\mathbf{b}]) / \partial a_{k}\right]^{2}\right.\right. \\
& \left.\left.\quad+\left[\partial f([\mathbf{a}],[\mathbf{b}]) / \partial b_{k}\right]^{2}\right\}\right) .
\end{aligned}
$$

This function is inserted into the original integral [Eq. (2.6)] for the time correlation function in both numerator and denominator to generate a DIS function analogous to that in Eq. (2.5). What one wants to search for then are the thermally accessible stationary (or near stationary) points in the $S_{-}$functional which lead to a $D_{0}$ value of 1.0 . These are the paths which contribute most in the evaluation of the path integral, as they give the largest values of the sampling function $\rho D_{0}$. In the next section we discuss a general method by which one can locate the stationary (or near stationary) points of the action functional.

\section{III.STEEPEST DESCENT AND SIMULATED ANNEALING METHODS TO SEARCH FOR EXTREMA IN THE ACTION}

In the previous section we outlined a general Monte Carlo method, the SPMC method, for evaluating quantum real time correlation functions at finite temperature. The sampling function used in this method, $\rho D_{0}$ or the dynamical importance sampling function, contains both thermal and dynamical information due to the $\rho$ and $D_{0}$ portions, respectively. The $\rho$ portion of the sampling function contains thermal information since it has the structure of the exponential of a Hamiltonian integrated along a path, as one would find in an equilibrium path integral calculation. The $D_{0}$ function, on the other hand, emphasizes portions of configuration space where the action (or phase function) is stationary and damps regions where the action is not stationary, and thus directs the sampling into the dynamically important regions of configuration space. The dynamically important regions are in fact the classical paths at times much longer than the thermal time. The $\rho$ and $D_{0}$ functions thus reflect different (and sometimes competing) properties of the system. By 
locating the stationary points of the thermal and action portions of the sampling function, one obtains both the important thermal and dynamical regions of the configuration space of the quantum system in contact with a finite-temperature heat bath. We describe two methods which enable one to locate the stationary points.

The first derivatives of the thermal and action functionals with respect to the Fourier coefficients used to parametrize the path are given by (see the Appendix)

$$
\frac{\partial S_{ \pm}([\mathrm{a}])}{\partial a_{k}}=\frac{a_{k}}{s_{k}^{2}} \pm \frac{\beta}{2} \int_{0}^{1} \frac{\partial V[x(u)]}{\partial x} \sin (k \pi u) d u \text {. }
$$

These first derivatives are calculated as the sine transform of the force along the path leading from $x$ to $x^{\prime}$. Once these first derivatives are calculated, they can be implemented in a steepest descent algorithm to drive the functionals $S_{+}$and $S_{\text {_ }}$ to the nearest minima, if indeed minima exist with respect to each of the Fourier coefficients. That is, we solve the set of differential equations

$$
\dot{a}_{k}=-\nabla S_{ \pm}(\mathrm{a})
$$

given a fixed $x$ and $x^{\prime}$, via some propagation method. The time derivative refers to motion along the path of steepest descent given by the gradient of the $S_{ \pm}$functional with respect to the Fourier coefficients. In our calculations we used a fourth-order Runge-Kutta algorithm to propagate the system of equations to the nearest minimum. The quenches were initiated at periodic, well-spaced intervals along the Metropolis walk. We will see below that only at relatively short physical times can one quench with respect to all of the coefficients to a local minimum in the action $S_{-}$.

The second optimization procedure we describe is a simulated annealing procedure to locate extrema of the action and minima of the thermal functional $S_{+}$. The annealing procedure utilizes the sampling function $\rho D_{0}$ to locate the extrema. Simulated annealing is a useful and general tool in combinatorial optimization problems, and it and related artificial intelligence methods have proved useful in many contexts. ${ }^{27}$ The actual function used in the annealing procedure is the DIS function employed in the Monte Carlo evaluation of the time correlation function:

$$
\begin{aligned}
F_{S}= & \left|\rho_{f p}\left(x^{\prime}, x, \beta_{c}\right)\right|^{2} \exp \left(-\left[S_{+}([\mathbf{a}])+S_{+}([\mathbf{b}])\right] / T_{1}\right) \\
& \times \exp \left(-\frac{1}{2 T_{2}} \sum_{k=1} \tau^{2} \epsilon_{k}^{2}\left\{\left[(\partial f[\mathbf{a}],[\mathbf{b}]) / \partial a_{k}\right]^{2}\right.\right. \\
& \left.\left.\left.+[\partial f[\mathbf{a}],[\mathbf{b}]) / \partial b_{k}\right]^{2}\right\}\right)
\end{aligned}
$$

The $\partial f / \partial a_{k}$ [written as $\left(S_{-}^{\prime}\right)$ below] and $S_{+}$functionals are described in Sec. II and the two "temperatures," $T_{1}$ and $T_{2}$, allow one to selectively anneal onto minima in the $S_{+}$ functional, the $\left(S_{-}^{\prime}\right)^{2}$ functional, or simultaneously onto minima in both. An exponential heating or cooling rate was employed in the annealing schedule, with the heating or cooling rate chosen slow enough so that the Metropolis Monte Carlo walk could find the minima of interest, but not so slow that the relaxation always proceeded to a global minimum. Heating or cooling in this context simply means ad- justing a parameter, $T_{1}$ or $T_{2}$, so as to drive the Metropolis Monte Carlo walk to a nearby minimum in the function of interest.

By cooling both pieces of the sampling function at the same rate, the system anneals onto paths which try to minimize both pieces simultaneously. That is, the system drifts towards paths which are nearly stationary in the action but yet have an appreciable thermal weight. On the other hand, if we heat the $S_{+}$portion of the sampling function while cooling the $\left(S^{\prime}\right)^{2}$ piece, the system will originally wander about thermally favorable portions of the Fourier coefficient space but ultimately drift to stationary regions of the phase function itself, i.e., extrema of the action.

If the stationary points are well isolated and the Metropolis walk has difficulty "finding" each stationary point during the course of the Monte Carlo simulation, the annealing procedure can be used to first heat the $\left(S^{\prime}-\right)^{2}$ portion of the sampling function and then rapidly cool onto local minima. By using the annealing procedure outlined above, one can get a good idea of the important parts of the configuration space and thus the sampling issues involved in the computation of the path integral.

Another important attribute of the annealing method is that it is capable of finding local minima in the functional $\left(S_{-}^{\prime}\right)^{2}$, where $\left(S_{-}^{\prime}\right) \neq 0$. These regions do not correspond to stationary points of the action but rather to locally slowly varying regions. These paths may be important in tunneling problems, where one may have complex roots to the set of nonlinear equations necessary to analytically determine the stationary phase points. The annealing procedure in this case locates the real portion of roots which themselves are not too far off the real axis. Since the path integral is calculated over a set of real variables, the annealing procedure in principle locates all stationary or locally slowly varying regions over the appropriate range of integration variables. The steepest descent procedure on the action does not locate such regions which are slowly varying relative to neighboring regions: Rather it locates the nearest stationary point in the action, if it exists. Using steepest descents to find the slowly varying regions would involve quenching on the $\left(S^{\prime}\right)^{2}$ function, which would necessitate the calculation of second derivatives of the action with respect to the Fourier coefficients.

To reiterate, the optimization method discussed above can be used to locate the important portions of configuration space in the evaluation of the path integral of Eq. (2.6) as determined by the DIS function $\rho D_{0}$. The steepest descent procedure is useful at short physical times when the action at the stationary points is a local minimum with respect to all of the Fourier coefficients (see below). Generally the type of path observed at short times is nearly a straight line path since the system in effect has little time to wander about the potential surface. One then expects to see one or at most a few stationary paths linking two end points. At longer physical times, two effects are observed. The action at stationary points can become locally maximal with respect to the loworder coefficients, and therefore the simulated annealing procedure is a more general tool which can be applied for all physical times to locate the extrema of the action by minimizing the functional $\left(S_{-}^{\prime}\right)^{2}$. Also, in general one observes 
a larger number of stationary points for anharmonic systems at long times since the path can explore larger portions of the potential surface. The Metropolis walk coupled with heating and cooling cycles in the annealing schedule leads to a thorough exploration of the configuration space which facilitates the location of the large number of extrema. The disadvantage of annealing is that it is slightly more costly than quenching, although both procedures require only modest amounts of computer time.

\section{RESULTS}

\section{A. Harmonic oscillator}

The quantum harmonic oscillator provides a good example of the utility of the quench-annealing procedure, since the stationary points are analytically available. However, the resulting set of equations is linear in each of the coefficients, so only a single stationary point exists for each set of end points $x$ and $x^{\prime}$. The set of equations for the stationary points is given by

$$
\frac{\partial S_{ \pm}([\mathbf{a}])}{\partial a_{k}}=\frac{a_{k}}{s_{k}^{2}} \pm \frac{\beta}{2} \int_{0}^{1} m \omega^{2} x(u) \sin (k \pi u) d u=0 .
$$

When the Fourier path is inserted in the integral, the following expression is obtained:

$\frac{\partial S_{ \pm}([\mathbf{a}])}{\partial a_{k}}=\frac{a_{k}}{s_{k}^{2}} \pm \frac{m \omega^{2} \beta}{2}\left(\frac{x}{k \pi}-\frac{x^{\prime}}{k \pi}(-1)^{k}+\frac{a_{k}}{2}\right)$.

Solving for each $a_{k}$ we get

$$
a_{k}=\left(\frac{m \omega^{2} \beta}{4} \pm \frac{1}{s_{k}^{2}}\right)^{-1}\left(\frac{m \omega^{2} \beta}{2 k \pi}\right)\left[x^{\prime}(-1)^{k}-x\right]
$$

for the stationary points of the action functional. The second derivatives are given by

$$
\frac{\partial^{2} S_{ \pm}(\mathbf{a})}{\partial a_{k}^{2}}=\left(\frac{1}{s_{k}^{2}} \pm \frac{m \omega^{2} \beta}{4}\right)
$$

Notice that for the equilibrium factor $S_{+}$the curvature is always positive so that quenches in this functional always lead to local minima. The curvature in the action functional, however, becomes negative under the condition

$$
\left(\pi^{2} k^{2}\right) /\left(\frac{\beta^{2}}{4}+\frac{t^{2}}{\hbar^{2}}\right) \hbar^{2}<\omega^{2}
$$

That is, as time increases, the stationary points of the action functional become local maxima with respect to the loworder coefficients. The singularity where the second derivative is zero is termed a caustic. For the harmonic oscillator, the caustics occur near times corresponding to the turning points in the classical motion.

The analytical results for a particular choice of end points $x$ and $x^{\prime}$ are presented in Table $I$. The number of Fourier coefficients $k_{\max }$ was chosen to be 7 throughout. The values of the coefficients are listed as a function of the coefficient number and the physical time in units of oscillator per-
TABLE I. Analytical results for the harmonic oscillator: the stationary points of the action functional at $t=0.4$ and $t=1.0$ oscillator periods, and the curvature for the $a_{k}$ set of coefficients.

\begin{tabular}{rrrrr}
\hline & $k$ & \multicolumn{1}{c}{$a_{k}$} & \multicolumn{1}{c}{$b_{k}$} & $a_{k}$ curvature \\
\hline$t=0.4$ & 1 & $0.182 e+00$ & $0.182 e+00$ & $0.101 e+01$ \\
& 2 & $-0.217 e-01$ & $0.217 e-01$ & $0.127 e+02$ \\
& 3 & $0.190 e-02$ & $0.190 e-02$ & $0.322 e+02$ \\
& 4 & $-0.232 e-02$ & $0.232 e-02$ & $0.594 e+02$ \\
& 5 & $0.389 e-03$ & $0.389 e-03$ & $0.945 e+02$ \\
& 6 & $-0.669 e-03$ & $0.669 e-03$ & $0.137 e+03$ \\
& 7 & $0.140 e-03$ & $0.140 e-03$ & $0.188 e+03$ \\
$t=1.0$ & 1 & $-0.842 e-01$ & $-0.842 e-01$ & $-0.218 e+01$ \\
& 2 & $0.387 e+01$ & $-0.387 e+01$ & $-0.713 e-01$ \\
& 3 & $0.178 e-01$ & $0.178 e-01$ & $0.345 e+01$ \\
& 4 & $-0.165 e-01$ & $0.165 e-01$ & $0.837 e+01$ \\
5 & $0.250 e-02$ & $0.250 e-02$ & $0.147 e+02$ \\
6 & $-0.409 e-02$ & $0.409 e-02$ & $0.224 e+02$ \\
7 & $0.831 e-03$ & $0.831 e-03$ & $0.316 e+02$ \\
\hline \hline
\end{tabular}

iods. The quench results are listed in Table II for one time which gives a positive curvature in the action for all of the Fourier coefficients. The quenches, initiated at well-spaced intervals along the Metropolis walk, lead directly to the correct stationary phase point. The path is shown in Fig. 2. When the time is increased beyond $t=0.5$, however, the quenches become unstable because of the negative curvature of the action with respect to the first Fourier coefficient.

\begin{tabular}{|c|c|c|c|}
\hline & $k$ & $a_{k}$ & $\boldsymbol{b}_{k}$ \\
\hline $\begin{array}{c}\text { Quenches at } t=0.4 \\
\text { on phase function }\end{array}$ & $\begin{array}{l}1 \\
2 \\
3 \\
4 \\
5 \\
6 \\
7\end{array}$ & $\begin{array}{r}0.182 e+00 \\
-0.217 e-01 \\
0.190 e-02 \\
-0.231 e-02 \\
0.387 e-03 \\
-0.664 e-03 \\
0.138 e-03\end{array}$ & $\begin{array}{l}0.182 e+00 \\
0.217 e-01 \\
0.190 e-02 \\
0.231 e-02 \\
0.387 e-03 \\
0.664 e-03 \\
0.138 e-03\end{array}$ \\
\hline $\begin{array}{l}\text { Simulated annealing } \\
\text { at } t=0.4 \text { on phase } \\
\text { function }\end{array}$ & $\begin{array}{l}1 \\
2 \\
3 \\
4 \\
5 \\
6 \\
7\end{array}$ & $\begin{array}{r}0.218 e+00 \\
-0.268 e-01 \\
0.309 e-02 \\
-0.160 e-02 \\
0.133 e-02 \\
0.156 e-02 \\
0.346 e-02\end{array}$ & $\begin{array}{l}0.173 e+00 \\
0.257 e-01 \\
0.174 e-02 \\
0.157 e-02 \\
0.235 e-02 \\
0.152 e-02 \\
0.151 e-03\end{array}$ \\
\hline $\begin{array}{l}\text { Simulated annealing } \\
\text { at } t=1.0 \text { on phase } \\
\text { function alone }\end{array}$ & $\begin{array}{l}1 \\
2 \\
3 \\
4 \\
5 \\
6 \\
7\end{array}$ & $\begin{array}{r}-0.871 e-01 \\
0.368 e+01 \\
0.161 e-01 \\
-0.138 e-01 \\
0.182 e-02 \\
-0.503 e-02 \\
0.144 e-02\end{array}$ & $\begin{array}{r}-0.852 e-01 \\
-0.386 e+01 \\
0.168 e-01 \\
0.185 e-01 \\
0.384 e-02 \\
0.291 e-02 \\
0.290 e-02\end{array}$ \\
\hline $\begin{array}{l}\text { Simulated annealing } \\
\text { at } t=1.0 \text { on thermal } \\
\text { and phase functions }\end{array}$ & $\begin{array}{l}1 \\
2 \\
3 \\
4 \\
5 \\
6 \\
7\end{array}$ & $\begin{array}{r}-0.655 e-01 \\
0.524 e-01 \\
0.836 e-03 \\
-0.163 e-02 \\
-0.285 e-02 \\
-0.599 e-02 \\
-0.211 e-02\end{array}$ & $\begin{array}{r}-0.736 e-01 \\
-0.382 e-01 \\
-0.384 e-03 \\
-0.231 e-02 \\
0.351 e-02 \\
-0.124 e-01 \\
-0.728 e-02\end{array}$ \\
\hline
\end{tabular}
Similarly, quenches with respect to each successive coeffi-

TABLE II. Quench and simulated annealing results for the harmonic oscillator at $t=0.4$ and $t=1.0$ periods. 


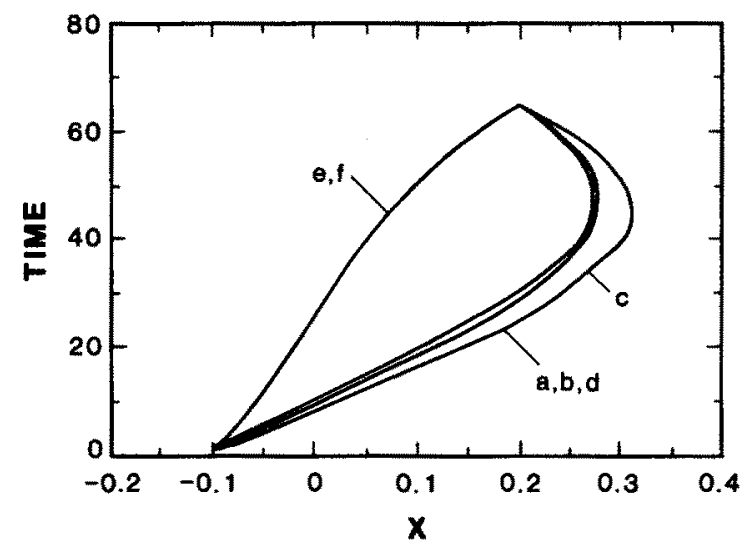

FIG. 2. Stationary paths for the harmonic oscillator at a short time, $t=0.4$ periods. The temperature is set to $500 \mathrm{~K}$ with an oscillator frequency of $\omega=1000 \mathrm{~K}$ and mass of $m=1.0 \mathrm{a}$.u. The time axis is number of discretization steps along a path. Distances are in a.u. Both quenches $(a, b)$ and simulated annealing $(c, d)$ were applied to the $S_{m}$ functional. Quenching and annealing lead to nearly identical paths. Also, a quench was performed on the $S_{+}$functional to determine the paths with the optimal thermal weight $(e, f)$. Notice that the stationary paths for the action and thermal pieces differ in this case. The thermal weight $\rho$ of the paths determined by quench-

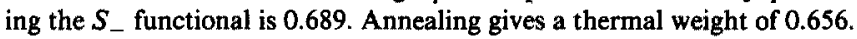
The thermal weight of the optimal $S_{+}$paths is 0.953 .

cient become unstable at the next half period of the oscillator.

The annealing procedure can also be used to locate the stationary points for the harmonic oscillator. At short times, the annealing procedure finds the same stationary phase regions as the quench results (Table II and Fig. 2). The paths resulting from simulated annealing are not identical with the analytical results and quench results because the annealing procedure was terminated prior to complete convergence. The annealing paths are very close to stationary, however, since $D_{0}=1.00$. The cooling process can be continued to achieve arbitrary accuracy. At times where the quenches lead to divergences in the first Fourier coefficient, i.e., $t>0.5$ periods, the annealing method finds the relevant local maxima in the action, as well as the minima, which exhibits the utility of the annealing method for the location of all of the extrema of the action, even at long physical times (Table II and Fig. 3).

An important issue in the sampling procedures becomes apparent, for example, at $t=1.0$, the point where the curvature in the action with respect to the second coefficient begins to take on a negative value. The analytically determined stationary point at $t=1.0$ has a very large second coeffcient, $a_{2}=3.87$. This large coefficient leads to large kinetic and potential energies in the equilibrium function $\rho$, which in turn leads to a very small thermal weight for this particular path. If the annealing is performed as a cooling process on both the thermal and action parts of the sampling function simultaneously, the system drifts towards a path for which the second coefficient is constrained to not move towards the large value of the stationary path due to the large kinetic and potential energies of such a path (Table II and Fig. 3). However, when the thermal portion of the sampling function is heated while the dynamical portion is cooled, the system is free to drift towards the true extrema of the action along the

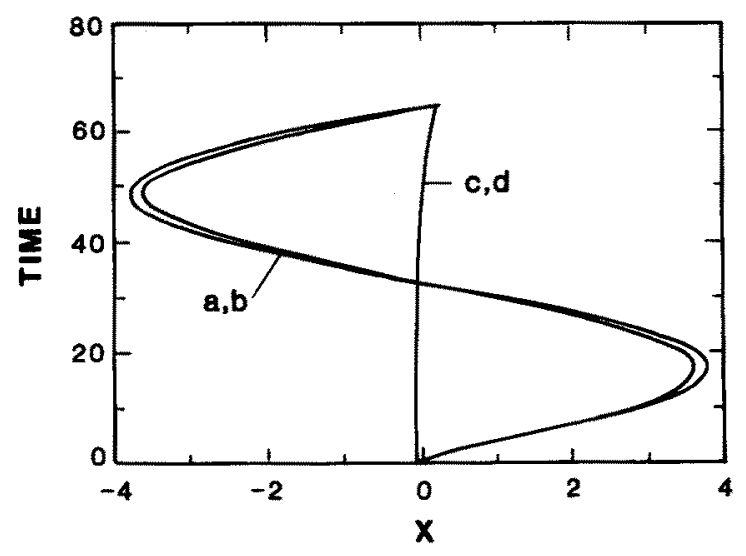

FIG. 3. Optimal paths for the harmonic oscillator at $t=1.0$ periods determined by simulated annealing. The stationary paths in the action $(a, b)$ give a $D_{0}$ value of 1.0 and lead to a thermal weight of $10^{-34}$. When annealing is applied to both pieces of the DIS function simultaneously, the more direct paths result $(c, d)$. For this case $D_{0}=0.951$ and $\rho=0.961$, which exhibits the tradeoff in optimizing both functions simultaneously.

course of the annealing procedure, and the large second coefficient is obtained. This indicates that there may be to some degree a "competition" between the equilibrium and dynamical pieces of the sampling function at physical times where caustics or near caustics occur.

The quench-annealing procedure is a useful tool, then, to locate stationary points in the multidimensional path integral expression for the harmonic oscillator time correlation function. Also, the annealing procedure gives insight into which classical paths are thermally accessible paths. The annealing procedure locates stationary paths at long physical times and at times where caustics or near caustics occur.

\section{B. Anharmonic systems}

It is apparent from Eq. (3.1) that, if the potential contains anharmonic terms, the path integral may exhibit multiple paths linking two end points in the configuration space which are stationary points in the action. The analytical calculation of the stationary points would involve solving a set of coupled $k_{\max }$ dimensional nonlinear equations, a nonlinear root finding procedure that would become very difficult in a high-dimensional system. The quench-annealing procedure provides a practical alternative tool to locate the stationary paths in these anharmonic systems. We choose three example anharmonic one-dimensional systems to search for multiple stationary phase behavior: a simple anharmonic oscillator, a Morse oscillator, and a double-well oscillator which exhibits tunneling behavior. The quench and simulated annealing procedures were used on these systems at various physical times. Multiple stationary phase points are found for all three cases.

The first example anharmonic system we examine is the harmonic oscillator plus an anharmonic term:

$$
V(x)=0.5 m \omega^{2}\left(x^{2}+0.4 x^{4}\right) .
$$

The effects of the anharmonicity on the time correlation function are discussed in Ref. 9. The oscillator frequency is chosen to be $\omega=1000 \mathrm{~K}$, the mass $m=1.0$ a.u., and the temperature $T=1000$ and $2000 \mathrm{~K}$. The quench procedure, 


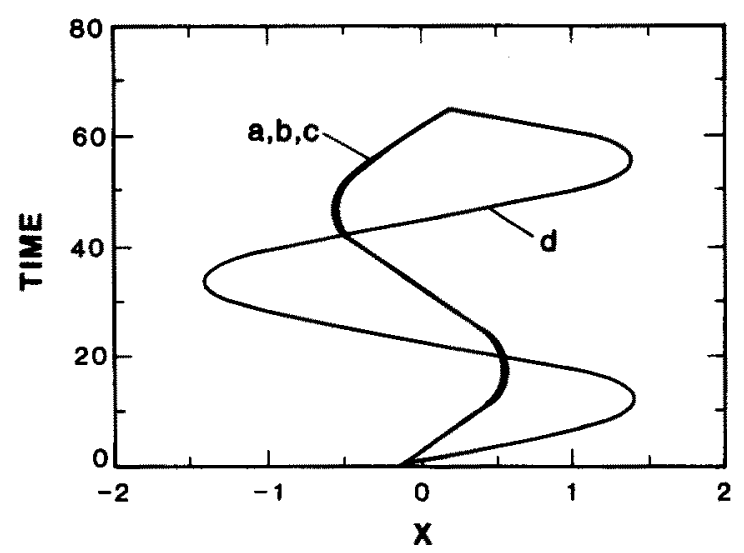

FIG. 4. Multiple stationary paths for the first anharmonic example with $T=1000 \mathrm{~K}$. The thermal weight of the paths with one period of oscillation $(a, b, c)$ is 0.37 . When the $a$ path has one oscillation period and the $b$ path 1.5 oscillations ( $c$ and $d$ ), the thermal weight is 0.0027 . At $T=2000 \mathrm{~K}$, the two paths are essentially superimposable on the paths at $T=1000 \mathrm{~K}$, indicating that the paths are temperature independent and thus classical paths.

at a time less than one-half period of the oscillator, $t=0.4$ periods, results in only one stationary point. However, at $t=1.0$, where quenches are unstable with respect to the loworder coefficients, the annealing procedure applied to the $\left(S_{-}^{\prime}\right)^{2}$ function alone (in what follows we apply the annealing procedure to the $D_{0}$ function alone to locate the stationary points of the action) leads to multiple stationary phase points. Some of the paths are shown in Fig. 4, along with the associated thermal weights. One path appears to dominate in that it gives the largest thermal weight. The second set of paths also is a stationary point but the larger displacement in the b path results in a much smaller thermal weight. Notice that the stationary paths are independent of temperature, which indicates that, at this physical time, the paths can be termed classical paths. The annealing procedure found a large number of other stationary paths with very small thermal weights (on the order of 0.001 ).

We examine a Morse oscillator as a second example, where

$$
V(x)=D_{e}\left(1-e^{-\alpha x}\right)^{2} .
$$

We set $D_{e}=40000 \mathrm{~K}$ and $\alpha=2.0$ a.u. ${ }^{-1}$, respectively. The ratio of the temperature to the dissociation energy was chosen as 0.175 , with a mass of $1.0 \mathrm{a}$.u. The annealing procedure applied at $t=1.0$ period (determined from the curvature in the potential at $x=0$ ) locates two stationary paths linking the end points $x=0.00$ a.u. and $x^{\prime}=0.15$ a.u. (Fig. 5). The two paths result in similar thermal weights. One path first moves up the steep repulsive part of the potential surface and then moves out and back in the $x$ direction before stopping at the end point. The second path moves directly up the longrange portion of the potential surface before returning to the end point $x^{\prime}$. The path which stays more confined to the region near the minimum in the potential surface has the favorable thermal weight. At any rate, at least two stationary paths are important in the Metropolis sampling of the integral, and interference effects due to the two paths are likely to be important.

Finally, we use the annealing procedure to locate multi-
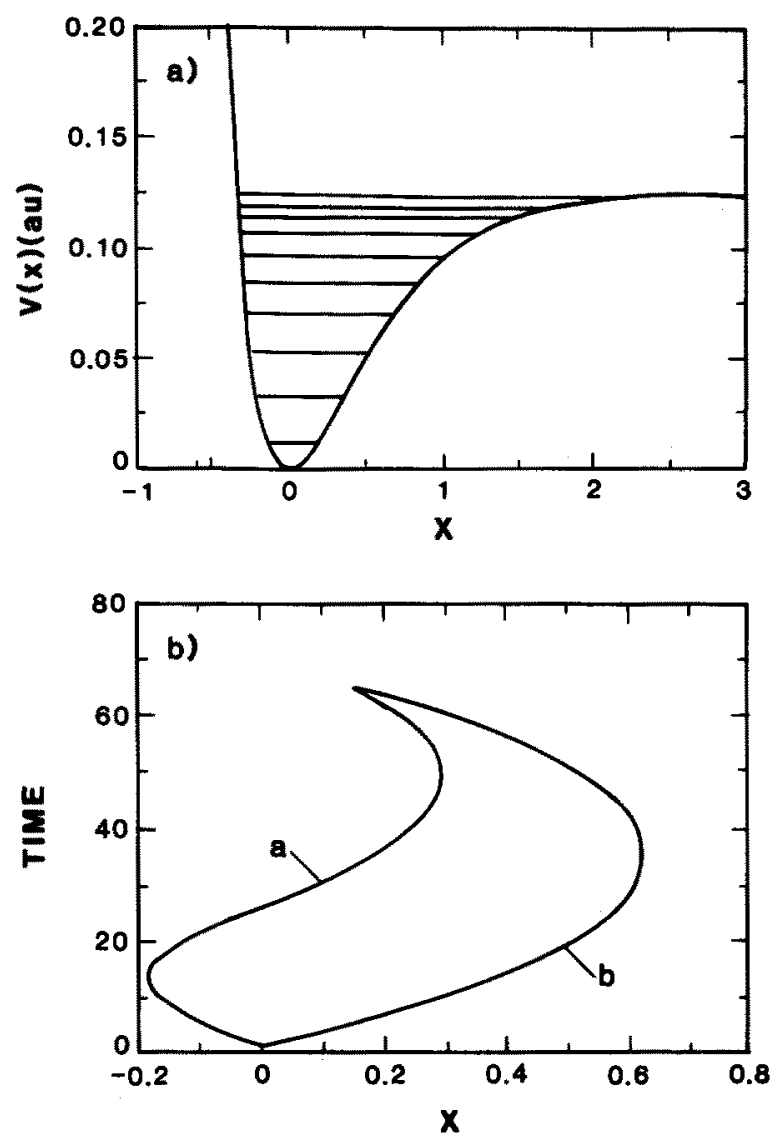

FIG. 5. Multiple stationary paths for the Morse oscillator. (a) The Morse potential function and eigenvalues, determined analytically. (b) Two stationary paths at $t=1.0$. If both $\mathbf{a}$ and $b$ paths are of the form of path $a$, the thermal weight is 0.314 . If the two paths are as shown ( $a$ and $b$ ), the thermal weight is 0.136 , i.e., both paths have an appreciable thermal weight, and interference effects from the two trajectories are likely to be important.

ple stationary phase (or near stationary) points in the action for a tunneling system. The potential we use is a harmonic well plus a Lorentzian barrier centered on the origin ${ }^{28}$ :

$$
V(x)=0.5 m \omega^{2} x^{2}+A /\left[1+(x / \alpha)^{2}\right] .
$$

The parameters are chosen to be $\omega=1895 \mathrm{~K}$, the barrier height $A=1.5 \hbar \omega$, and the barrier width $\alpha=0.09$ a.u. The exact eigenvalues can be determined numerically for this one-dimensional example. The eigenvalues are shown in Fig. 6 and clearly indicate a tunneling system. The tunnel splitting for the parameters chosen is $1295 \mathrm{~K}$. The tunneling behavior is most pronounced at low temperatures which just allow excitation to the first excited state. In this case, the dipole autocorrelation function oscillates as a cosine wave with the frequency of the tunnel splitting. One might expect that at low enough temperatures, few if any classical paths would lead from one side of the barrier to the other.

We performed the annealing procedure at $T=500 \mathrm{~K}$ and $t=1.0$ periods (of the unperturbed oscillator) with end points first on the same side of the barrier and then on opposite sides. The annealing procedure always finds a stationary point with an appreciable thermal weight when the two end points are on the same side of the barrier [Fig. 6(b) ]. Clearly in this case the thermal energy necessary to "find" the other end point is not terribly large, and a classical path can 

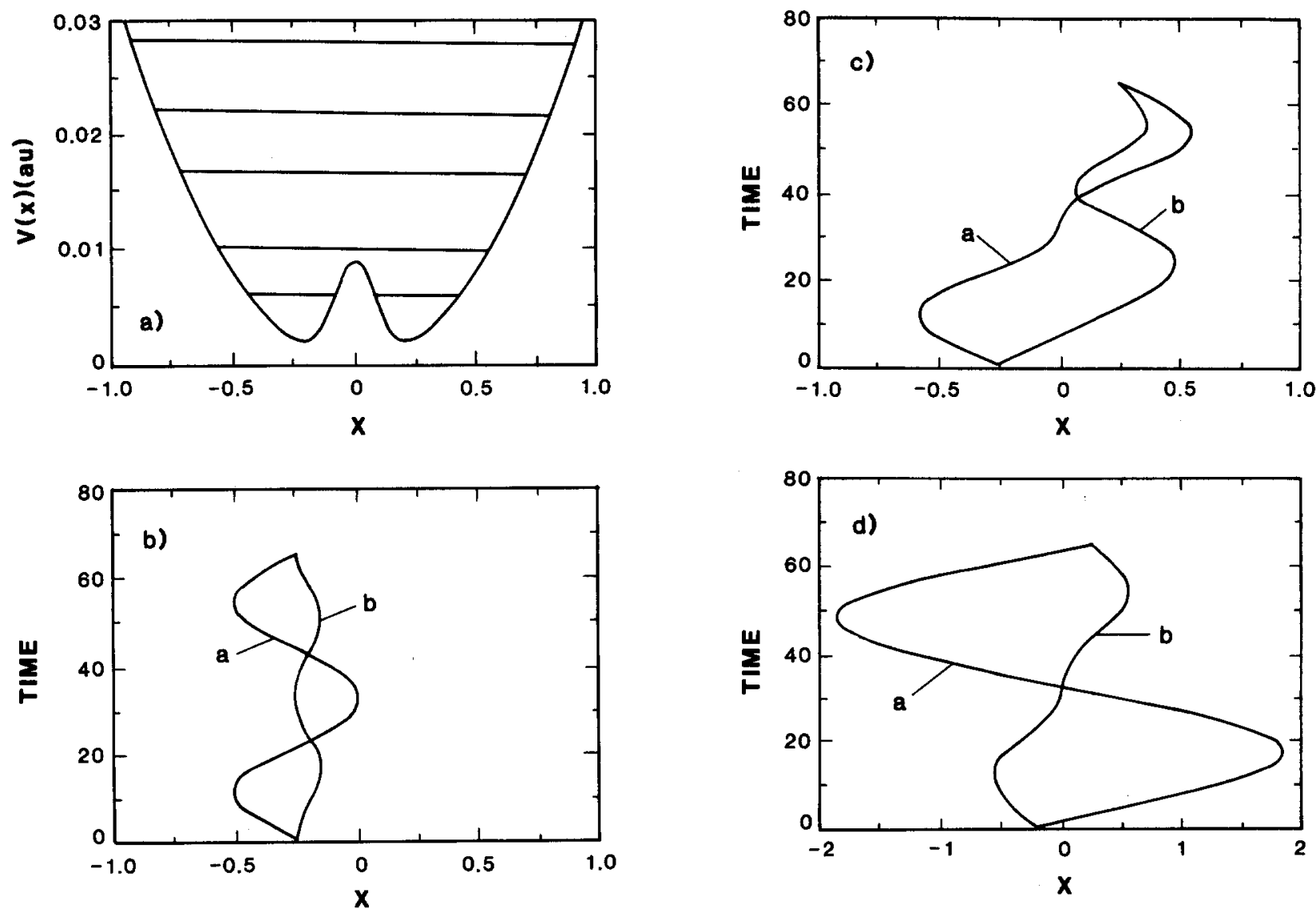

FIG. 6. Multiple stationary and near stationary paths for a tunneling system. (a) The double-well potential and eigenvalues. (b) Stationary paths with end points on the same side of the barrier: $\rho=0.026, D_{0}=1.00$. (c) End points on opposite sides of the barrier: $\rho=0.0057, D_{0}=0.964$. The a path is the single stationary path with an appreciable thermal weight. The b path is a near stationary or tunneling path. (d) $\rho=2.8 \times 10^{-16}, D_{0}=1.00$. The large amplitude of the a path leads to a small thermal weight. Both $\mathbf{a}$ and $\mathbf{b}$ paths are stationary.

be located which links the end points. Also, a large number of other stationary points with relatively small thermal weights are found.

When the annealing procedure is used to locate stationary points when $x$ and $x^{\prime}$ are located on separate sides of the barrier, however, it locates local minima in the $\left(S_{-}^{\prime}\right)^{2}$ function in the majority of cases. These local minima correspond to regions where $\left(S_{-}^{\prime}\right) \neq 0$, that is locally slowly varying but not stationary points of the action [Figs. $6(\mathrm{c})$ and 6(d) ]. This behavior is indicative of tunneling paths linking the end points. A large number of local minima were found with similar, appreciable thermal weights, which points out the sampling difficulty for this particular problem. One stationary path was also observed with a thermal weight of on the order $\rho=0.0039$. A second stationary path was found which gives a $\rho$ value of $10^{-30}$. This implies that, for this case, only one thermally allowed stationary path exists, and the near stationary, tunneling paths give important contributions to the integral. Other stationary paths besides the thermally allowed path appear to be very much on the fringes of the Boltzmann distribution. Notice that the single stationary path has a "kink" in it near the point where the trajectory passes the barrier separating the two minima. The paths which result in a $D_{0}$ value of less than 1.0 appear to pass rapidly through the barrier region.

A simple one-dimensional example serves to illustrate the sampling issues that arise in a tunneling system. Consider a one-dimensional integral of the form of $\mathrm{Eq}$. (2.1) with the following phase function:

$$
f(x)=x^{4} / 4+x^{3} / 3+c x^{2} / 2 \text {. }
$$

The stationary points of this function occur at $x=0$, $[-1 \pm \sqrt{(1-4 c)}] / 2$. The roots are thus real or complex depending on the value of $c$. The function $D_{0}$ at various values of $c$ is presented in Fig. 7. Notice that the three stationary phase points are apparent when the roots are purely real. When $c>0.25$, however, two of the roots move off the real axis. The effect on $D_{0}$ is apparent in Fig. 7 as a shoulder in the distribution function at $x=-0.5$. The shoulder becomes less pronounced as the imaginary portion of the roots becomes larger. Notice that when $c=0.30, D_{0}$ has a local maximum at $x=-0.5$ with a value less than 1 . This local maximum occurs when the imaginary part of the complex root is not far off the real axis.

The $D_{0}$ function naturally weights paths which are stationary or near stationary in the action. The Monte Carlo integration of the path integral as described above occurs along the real axis, so all of the tunneling effects are in principle incorporated by sampling the appropriate portions of configuration space as determined by the DIS function, that is sampling all of the stationary or near stationary yet ther- 

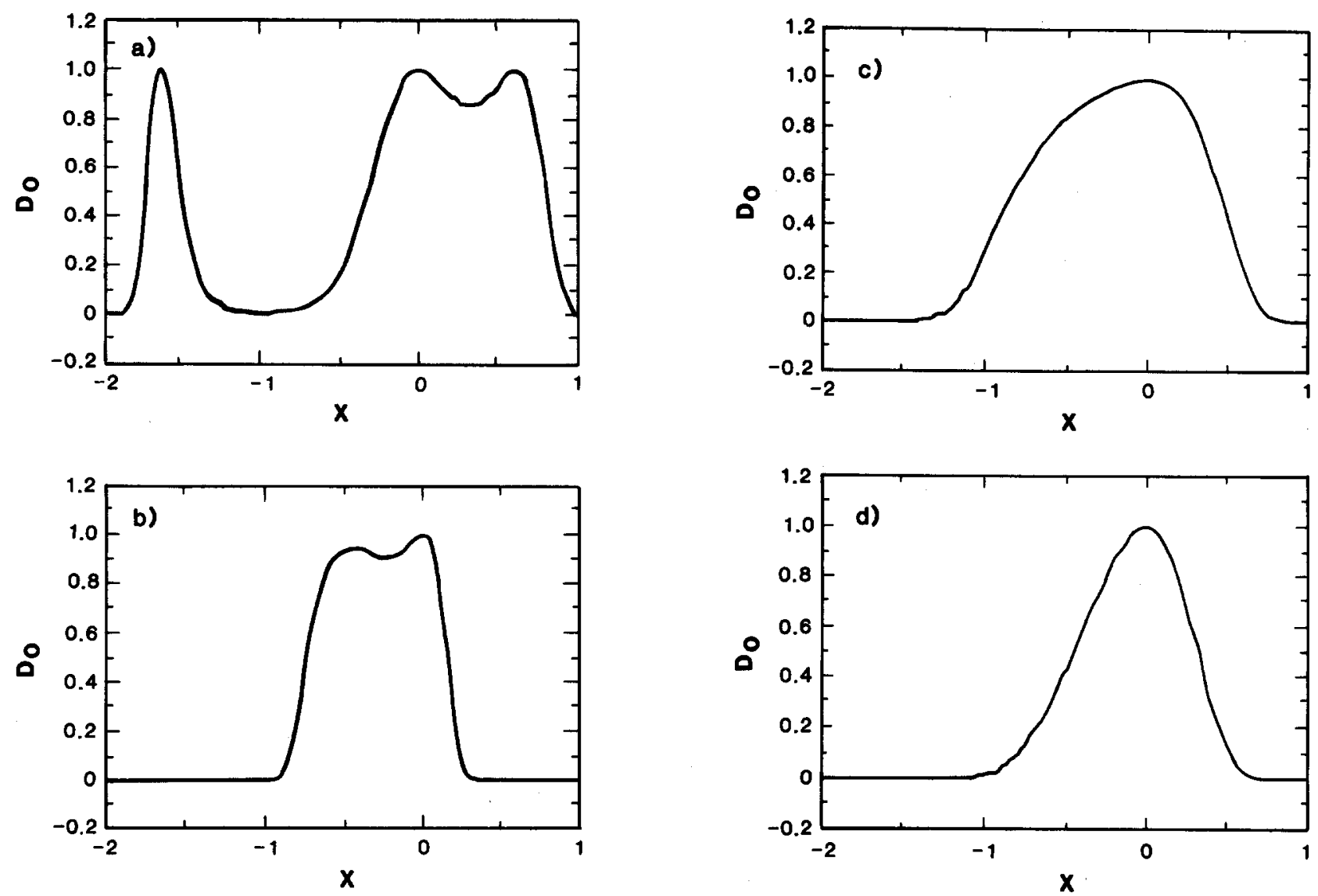

FIG. 7. $D_{0}$ for the one-dimensional example with complex roots: $f(x)=x^{4} / 4+x^{3} / 3+c x^{2} / 2$. (a) $c=-1.0$, (b) $c=0.30,(c)=1.0$, (d) $c=2.0$. Notice the local maximum in (b) at $x=-0.5$ with $D_{0}<1.0$.

mally accessible paths. However, it may be more efficient to allow the contour of the integration to distort to incorporate the complex valued tunneling trajectories. This idea was useful in certain barrier crossing problems where a coordinate rotation method was used, ${ }^{7,11}$ and has been discussed in other contexts. ${ }^{29}$

\section{DISCUSSION}

In this paper we have presented a general algorithm for locating stationary points in the phase function of multidimensional oscillatory path integrals which requires only first derivatives of the action. The stationary paths are the classical paths linking two end points over a time interval $t$ if $t \gg \beta \hbar$. The method involves a combination of steepest descent and simulated annealing procedures. It is found to effectively locate the single stationary phase point for the harmonic oscillator given a set of path end points. Also, the quench-annealing procedure locates and assigns a thermal weight to multiple stationary paths in nonlinear problems, however anharmonic the potential function. This information is useful in itself as it gives some indication of the important portions of configuration space and thus of the complexity involved in a Metropolis Monte Carlo sampling strategy.

It may also be useful when formulating, for example, a semiclassical theory with thermal averaging in very anharmonic systems to know the classical or stationary paths which contribute appreciably to the physical process of interest. This information determines the degree of phase interference that must be included from separate classical paths in the thermally averaged semiclassical picture.

For a system in which classically forbidden paths dominate the process of interest, the annealing procedure as described above locates paths which are not strictly stationary in the action, but which are locally slowly varying. The lack of stationary paths linking the two points on opposite sides of the barrier at low temperatures is indicative of this tunneling behavior. Only trajectories very much on the fringe of the Boltzmann distribution can classically connect two end points on opposite sides of the barrier, if the temperature is small relative to the barrier height. Also, in a multidimensional tunneling system, the annealing procedure can be used to determine whether there exists any classically allowed paths which link two end points.

Once the path integral for the time-dependent quantity is written as in Eq. (2.6), the calculation of the multidimensional integral becomes a problem in sampling appropriately over the important portions of the configuration space. For a simple harmonic oscillator, where only one stationary phase point exists, the sampling is straightforward. However, when multiple stationary phase points exist in the problem, the sampling issue becomes formally similar to problems encountered in studying a classical system with a large number of local minima in the potential surface; in the dynamics 
problem the analogy of potential minima is minima in the function $\left(S_{-}^{\prime}\right)^{2}$. In the case of the classical system, the problem is one of locating all or most of the local minima in the potential surface which contribute appreciable Boltzmann weights to a given state and then devising a sampling scheme to allow the Metropolis walk to sample all of these regions. If $k T$ is small relative to the barriers separating the local minima, move strategies must be incorporated to allow infrequent hops between the local minima.

The simplest strategy is to first "catalogue" the set of stationary phase points and then incorporate into the move strategy the possibility of infrequent additions of vectors which connect the various stationary points or "lattice sites." These moves must be chosen randomly to ensure detailed balance in the Metropolis procedure. For example, a random choice of a "lattice vector" connecting two of the stationary phase regions could be added to the current configuration during a certain percentage of the moves. This procedure ensures detailed balance since the probability of attempting a move back to the original configuration is the same as for the trial move, and ensures that the relevant portions of configuration space are sampled. An example of this kind of rare event problem occurs in the study of surface diffusion at low temperatures, where a similar sampling strategy proved useful. ${ }^{30}$

Finally, it would be useful to utilize a more efficient strategy which incorporates the information obtained from the optimization procedures discussed above into the stationary phase Monte Carlo method. Staging ${ }^{31}$ and multigrid $^{32}$ methods may be useful in employing the large amplitude, collective motions necessary to connect the stationary phase regions in a practical way. Efforts are currently underway to utilize these Monte Carlo strategies.

\section{ACKNOWLEDGMENTS}

We would like to thank L. Pratt and G. Hoffman for helpful discussions. One of us (D.L.F.) acknowledges the donors of the Petroleum Research Fund, administered by the American Chemical Society for partial support of this work. D.L.F. also acknowledges support in part by a grant from the Capital Research Corporation.

\section{APPENDIX}

We consider here the Fourier path integral expression for the ratio of the general complex temperature density matrix element to its free particle counterpart,

$$
\begin{aligned}
& \frac{\left\langle x^{\prime}\left|e^{-\beta_{c} H}\right| x\right\rangle}{\left\langle x^{\prime}\left|e^{-\beta_{c} H}\right| x\right\rangle_{\mathrm{fp}}} \\
& \quad=\frac{\int d \mathbf{a} \exp \left(-\Sigma_{k=1} a_{k}^{2} / 2 \sigma_{k}^{2}-\beta_{c}\langle V\rangle\right)}{\int d \mathbf{a} \exp \left(-\Sigma_{k=1} a_{k}^{2} / 2 \sigma_{k}^{2}\right)},
\end{aligned}
$$

where $\beta_{c}$ is the complex temperature, and where the remaining quantities are given by

$$
\langle V\rangle=\int_{0}^{1} d u V[x(u)],
$$

$$
\begin{aligned}
& x(u)=x+\left(x^{\prime}-x\right) u+\sum_{k=1} a_{k} \sin (k \pi u), \\
& \sigma_{k}^{2}=2 \beta_{c} \hbar^{2} / m \pi^{2} k^{2} .
\end{aligned}
$$

We denote the exponent in the numerator of Eq. (A1) as

$$
S(\mathbf{a})=\sum_{k=1} a_{k}^{2} / 2 \sigma_{k}^{2}+\beta_{c}\langle V\rangle
$$

The requirement that $S$ be an extremum with respect to the Fourier coefficients is

$$
\frac{\partial S(\mathbf{a})}{\partial a_{k}}=0 \quad \text { all } k
$$

From Eqs. (A2)-(A5) it is straightforward to show that the condition that $S$ be an extremum becomes

$$
a_{k}=\frac{1}{2} \sigma_{k}^{2} \beta_{c} f_{k}(\mathbf{a}),
$$

where $f_{k}(\mathbf{a})$ is the $k$ th Fourier sine component of the force evaluated along the quantum-mechanical path specified by (A3):

$$
f_{k}=2 \int_{0}^{1} d u\left(-\frac{\partial V[x(u)]}{\partial x(u)}\right) \sin (k \pi u) .
$$

Equations (A7) and (A8) are a set of self-consistent conditions that determine the extrema of the path-integral "action" for general complex temperatures $\beta_{c}$. Knowledge of such extrema is a convenient starting point for various semiclassical approximations to the exact path-integral results.

Two special cases of the general results are noteworthy. If $\beta_{c}$ is purely real, $\beta_{c}=\beta$, then Eq. (A7) becomes

$$
a_{k}=\frac{\beta^{2} \hbar^{2}}{m \pi^{2} k^{2}} f_{k}(\mathbf{a}) \text {. }
$$

If, on the other hand, $\beta_{c}$ is purely imaginary, $\beta_{c}=i t / \hbar$, then Eq. (A7) becomes

$$
a_{k}=-\frac{t^{2}}{m \pi^{2} k^{2}} f_{k}(\mathbf{a}) \text {. }
$$

Planck's constant does not appear in Eq. (A10) since the result is a purely classical one: solution(s) to Eq. (A10) combined with Eq. (A3) describe the classical mechanical path(s) that travel from $x$ to $x^{\prime}$ in a time $t$. Building the desired initial and final end points of the classical path into Eqs. (A10) and (A3) may prove a useful alternative to traditional trajectory methods for various semiclassical applications.

'N. Metropolis, A. W. Rosenbluth, M. N. Rosenbluth, A. H. Teller, and E. Teller, J. Chem. Phys. 21, 1087 (1953). For a recent review, see J. P. Valleau and S. G. Whittington, in Modern Theoretical Chemistry, edited by B. J. Berne (Plenum, New York, 1977), Vol. 5, pp. 137-168.

${ }^{2}$ R. P. Feynman and A. R. Hibbs, Quantum Mechanics and Path Integrals (McGraw-Hill, New York, 1965).

${ }^{3}$ See, for example, Path Integrals and Their Applications in Quantum, Statistical, and Solid State Physics (Plenum, New York, 1978).

${ }^{4}$ For a cross section of recent activity in this area, see J. Stat. Phys. 43, 729 (1986).

${ }^{5}$ J. D. Doll, J. Chem. Phys. 81, 3536 (1984).

${ }^{6} \mathrm{~J}$. D. Doll and D. L. Freeman, Report No. LA-UR (86-4177); Adv. Chem. Phys. 73, 289 (1988).

${ }^{7}$ J. D. Doll, R. D. Coalson, and D. L. Freeman, J. Chem. Phys. 87, 1641 (1987).

'J. D. Doll, D. L. Freeman, and M. J. Gillan, Chem. Phys. Lett. 143, 277 (1988). 
${ }^{9}$ J. D. Doll, T. L. Beck, and D. L. Freeman, J. Chem. Phys. 89, 5753 (1988).

${ }^{10}$ V. S. Filinov, Nucl. Phys. B271, 717 (1986).

"J. Chang and W. H. Miller, J. Chem. Phys. 87, 1648 (1987).

${ }^{12}$ N. Makri and W. H. Miller, Chem. Phys. Lett. 139, 10 (1987).

${ }^{13}$ N. Makri and W. H. Miller, J. Chem. Phys. 89, 2170 (1988).

${ }^{14}$ R. E. Cline, Jr. and P. G. Wolynes, J. Chem. Phys. 88, 4334 (1988).

${ }^{15}$ R. Serra, M. Andretta, M. Compiani, and G. Zanarini, Introduction to the Physics of Complex Systems (Pergamon, New York, 1986).

${ }^{16}$ See, for example, L. R. Pratt, J. Chem. Phys. 85, 5045 (1986); R. Elber and M. Karplus, Chem. Phys. Lett. 139, 375 (1987); R. S. Berry, H. L. Davis, and T. L. Beck, ibid. 147, 13 (1988).

${ }^{17}$ L. S. Schulman, Techniques and Applications of Path Integration (Wiley, New York, 1981).

${ }^{18}$ P. Pechukas, Phys. Rev. 181, 166 (1969).

${ }^{19}$ E. J. Heller, J. Chem. Phys. 62, 1544 (1975); 64, 63 (1976).

${ }^{20}$ D. Huber and E. J. Heller, J. Chem. Phys. 87, 5302 (1987).

${ }^{21}$ W. H. Miller and T. F. George, J. Chem. Phys. 56, 5668 (1972).

${ }^{22}$ T. F. George and W. H. Miller, J. Chem. Phys. 57, 2458 (1972).

${ }^{23}$ C. W. McCurdy and W. H. Miller, J. Chem. Phys. 73, 3191 (1980).
${ }^{24}$ S. Wandzura, Phys. Rev. Lett. 57, 2603 (1986).

${ }^{25}$ J. R. Klauder, Phys. Rev. Lett. 56, 897 (1986).

${ }^{26}$ P. Zhang, R. M. Levy, and R. A. Freisner, Chem. Phys. Lett. 144, 236 (1988).

${ }^{27}$ W. H. Press, B. P. Flannery, S. A. Teukolsky, and W. T. Vetterling, $\mathrm{Nu}-$ merical Recipes (Cambridge, New York, 1986); E. H. L. Aarts and P. J. M. van Laarhoven, in Pattern Recognition Theory and Applications, edited by P. A. Devijver and J. Kittler (Plenum, New York, 1987); M. Eigen, in Emerging Synthesis in Science, edited by D. Pines (Addison-Wesley, New York, 1988); S. Kirkpatrick, C. D. Gelatt, Jr., and H. P. Vecchi, Science 220, 671 (1983); S. M. Lederman and R. A. Marcus, J. Chem. Phys. 88, 6312 (1988).

${ }^{28}$ D. L. Freeman and J. D. Doll, J. Chem. Phys. 80, 5709 (1984).

${ }^{29}$ D. W. McLaughlin, J. Math. Phys. 13, 1099 (1972).

${ }^{30}$ A. F. Voter and J. D. Doll, J. Chem. Phys. 80, 5814 (1984); A. F. Voter, ibid. 82, 1890 (1985).

${ }^{31}$ M. Sprik, M. L. Klein, and D. Chandler, Phys. Rev. B 31, 4234 (1985).

${ }^{32}$ D. Kandel, E. Domany, D. Ron, A. Brandt, and E. Loh, Jr., Phys. Rev, Lett. 60, 1591 (1988). 
The Journal of Chemical Physics is copyrighted by the American Institute of Physics (AIP). Redistribution of journal material is subject to the AIP online journal license and/or AIP copyright. For more information, see http:/ojps.aip.org/jcpo/jcpcr/jsp Copyright of Journal of Chemical Physics is the property of American Institute of Physics and its content may not be copied or emailed to multiple sites or posted to a listserv without the copyright holder's express written permission. However, users may print, download, or email articles for individual use. 
The Journal of Chemical Physics is copyrighted by the American Institute of Physics (AIP). Redistribution of journal material is subject to the AIP online journal license and/or AIP copyright. For more information, see http://ojps.aip.org/jcpo/jcper/jsp 